(Aus der Psychiatrisch-neurologischen Universitätsklinik in Budapest [Direktor Hofrat Prof. Dr. E. E. Moravesik].)

\title{
Beitrag zur Histopathologie und Bakteriologie der Chorea infectiosa.
}

\author{
Von
}

\author{
Dr. Julius Schuster,
}

I. Assistenten der Klinik.

Mit 14 Textabbildungen.

(Eingegangen am 14. Juni 1920.)

Veröffentlichungen über anatomische Veränderungen im Zentralnervensystem Choreakranker, liegen aus den früheren Zeiten in großer Anzahl vor, aber je schärfer man aus der großen Krankheitsgruppe, die mit choreiformen Bewegungsstörungen einhergeht, die infektiöse und die ihr völlig verschiedene Chorea Hungtingtoni heraushob, um so spärlicher wurden gerade bei ersterer positive pathologisch-anatomische Befunde.

Das Bestreben, den Sitz der eigentümlichen Erkrankung festzustellen ist alt, denn es fehlt bis jetzt an typischen, beständigen Veränderungen, die nur der Chorea infectiosa zukommen. Wenn wir die Literatur nach Befunden, die an Gehirn und Rückenmark von Choreakranken erhoben wurden, durchsehen, bietet sich ein ziemlich mannigfaches Bild verschiedener Veränderungen des Gefäßsystems, der Nervensubstanz und des Bindegewebes dar. 1869 beschreibt Broadbent im Thalamus und Nucleus lenticularis, capillare Thrombosen und baut seine embolische Theorie der Chorea auf seine Befunde auf. Me y nert fand einen Herd in der inneren Kapsel. Clark Körnchenzellen um die Gefäße, Jacks on capilläre Embolien. Schwarz Herde in der inneren Kapsel und Thalamus. Nach Dana sollen Gefäßveränderungen, wie umschriebene Erweiterungen der Gefäße, Degenerationen der Gefäßwand, erweiterte perivasculäre Lymphräume vorliegen. Elischer sah 1874 in Corpus striatum Gefäßverdickungen um die Capillaren; Pigmentschollenanhäufungen und konzentrisch geschichtete Körperchen. Nachdem Jakowenka die Choreakörperchen gesehen hatte und Wollenberg die Nichtspezifität der Körperchen, indem er dieselben bei Nichtchoreatikern nachwies, aussprach, tritt Hudovernig wieder für die Spezifität der Choreakörperchen ein. Blutungen beschreiben Ogle, Dickinson, Thomson, Elischer, Nauwerck, Handford, Powell, Reichardt, Cramer und Többen, Tommasi, Coudeli, Wollenberg und Sand. Pons, Medulla oblongata, Hirnrinde, Marklager waren betroffen. Regellose Verteilung capillärer Thrombosen beschreiben Handford, Okada, Poynton und Holmes, Donath, Oppenhei m. Perivasculäre Infiltrationen der Gefäßwände selbst und mehr oder weniger von Gefäßen abhängige Erweichungsherde sind in der Literatur bei Litten, 
Nauwerck, Ogle, Welleminsky, Pianese, Reichardt, Hudovernig Poynton und Holmes, Orsechowsky, Tomasi - Coudeli, Abrahams, L épine, Girand, Rebattue, Sand erwähnt. Auch diese Beschreibungen geben keine bestimmte Lokalisation an. Gliaveränderungen finde ich beschrieben bei Cramer und Többen, Wendenburg, Poynton und Holmes, Orzechows ki, Sand und Alzhei mer. Fettige und pigmentige Entartung der Ganglienzellen (Elischer, Sachs, Little, Reichardt). Poynton und Holmes, Sachs, Sand beschreiben die Tigrolysis und Chromatolysis der Ganglienzellen. Wenn wir die Deutung der Befunde der Chorea infectiosa durchsehen, so äußern sich immer mehr und mehr Forscher etwas zurückhaltender. Z. B. Elischer bringt die Ganglienzellendegenerationen im Marklager, die Blutungen, die Eiterherde, die sich um die Gefäße fanden, mit dem puerperalen Prozeß in Verbindung, der die Kranke erlag. O ka da möchte die mikroskopischen multiplen Thrombosen im motorischen Gebiet des Großhirns nicht unbedingt für die Erzeugung der Chorea verantwortlich machen. Die inkonstanten pathologisch-histologischen Befunde seien unter keinen Umständen als das anatomische Substrat der Chorea anzusehen, meint Köster. Rindfleisch nimmt an, daß die Befunde von Komplikationen, von pyämischen Prozessen, Encephalitis, Endokarditis, Rheumatismus der Gelenke bei Chorea von derartigen embolischen und encephalopathischen Prozessen herrühren. Orzechowski bringt die Abweichungen von der Norm bei Chorea in Beziehung zu der Septicopyämie, an welcher diese Kranken meist sterben. Nur in den sehr schweren Fällen, die in einigen Tagen sterben, könnte man rein choreatische Veränderungen finden. Diese Fälle geben bei der Autopsie ein negatives Resultat. Wendenburg ist der Meinung, daß die embolischen, entzündlichen und thrombotischen Prozesse im Zentralnervensystem mit der Chorea direkt nichts zu tun haben, es seien nur Zeichen einer Sepsis, die Ursachen der Verschlimmerung des septischen Grundleidens der Chorea. Die negativen Fälle bei letaler Chorea werden als wichtiges Argument gegen die Spezifität der Gewebsveränderungen der Chorea infectiosa betrachtet.

Reinhold beschrieb eine Sinusthrombose, ohne besondere bezeichnende Veränderungen im Gehirn, ebenso Triboulet bei einem Kranken, der an Pericarditis und Chorea litt. Werner fand bei an Endokarditis und Nephritis parenchymatosa Gestorbenen im Gehirn und Rückenmark nichts Bezeichnendes. Rindfleis ch konnte bei seinen Fällen (Chorea minor mit Endokarditis und Perikarditis und lobuläre Pneumonie) im Gehirn außer massiger Erweiterung der perivasculären und pericellulären Lymphräume im Thalamus und Linsenkern und verlängerten Mark starke Aufquellung der Nissl-Schollen an den Rückenmarkvorderhornzellen nichts Spezifisches nachweisen. Do nath konstatierte ein thrombosiertes Gefäß in seinem Fall, keine sonstigen histologischen Veränderungen.

Den Gehirnbefunden bei Chorea minor stehen als Gegenstück die Befunde im Zentralnervensystem von septischen und Infektionskrankheiten gegenüber und bieten wegen des Vergleichs bei dem Befunde viel Interessantes.

Außer Popoff hatten Blaschko und Savade, die die sichtlich unveränderten Gehirne Typhuskranker untersuchten, in den perivasculären und periganglionären Räumen Lymphocyten in großer Anzahl gefunden, weiter sah Blaschko bei experimenteller Sepsis Kokkenemboli in Capillaren und Venen zerstreut. Hyperämie, Blutextravasate, Leukocyteninfiltration der weichen Gehirnhäute bei einem Fall von Sepsis wurde 1901 von Savade beobachtet. E. Fraenkel fand sehr oft bei verschiedenen Infektionskrankheiten Bakterien. 
So wurden 85 Gehirne von an verschiedenen Infektionskrankheiten Verstorbenen untersucht. Ansammlung von Pigmentschollen, Thrombenbildung, Proliferationserscheinungen in den Meningen und Blutungen, Nekrosen in der Gehirnsubstanz sind Zeichen der intravitalen Entstehung der Prozesse. 1912 beschrieb Oseki ähnliche Befundé im Gehirn bei Infektionskrankheiten. Nekroseherde und Bakterienhaufen im Mark und Rinde, umschriebene Leukocytenansammlungen, Meningoencephalitis. Polymorphkernige Leukocyten und Lymphocyten in den perivasculären Lymphräumen.

Wie ersichtlich, ist die Lokalisation der choreatischen Veränderungen noch nicht gelungen. Es seien nun die Krankengeschichtenauszüge von mit Tod ausgegangenen vier Choreafällen, die ich in der psychiatrischneurologischen Universitätsklinik in Budapest $\mathrm{zu}$ beobachten und zu untersuchen Gelegenheit hatte, in Kürze mitgeteilt.

I. Der erste Fall betrifft eine 19 Jahre alte Lehrerin E. K., die mit schweren, heftig einsetzenden choreatischen Bewegungsstörungen und Fieber in die psychiatrisch-neurologische Universitätsklinik am 15. August 1911 aufgenommen wurde und dort nach 6 Tagen an Erschöpfung und Sepsis starb. Bei der Aufnahme hatte die zart gebaute, schwach entwickelte, sehr blutarme Kranke im ganzen Körper choreatische Bewegungen von großer Heftigkeit, welche bei jeder psychischen Erregung zunahmen.

Am 16. Aug. 1911 wurden $10 \mathrm{ccm}$ Blut aus der Vena mediana dextra entnommen und in 6 Serumbouillonröhrchen zerteilt, bei $37^{\circ} \mathrm{C}$ im Thermostat belassen. Es entstand eine feine Trübung. Aus den Röhrchen werden Serumagarplatten beschickt und ein Teil des Blutes wurde in Serumagarplatten gegossen. In 24 Stunden wuchsen zarte, gelbliche Kolonien, der Staph ylococcus pyogenes a ure us. Das Gehirn wurde 2 Stunden nach dem Tode durch die Carotiden mittels 10 proz. Formalinlösung ausgespritzt und so fixiert. Obduktion am 22. Aug. 1911 (Dozent Dr. Béla Entz).

Aus dem Obduktions befund: In den Kammern und Vorhöfen des Herzens wenig flüssiges und halbgeronnenes Blut. Die Klappen der Aorta, der Lungenschlagader und der dreiteiligen Klappe dünn, membranös. An der inneren Fläche der Bicus pidalklappen in der Insertionslinie sind feine, a us durchscheinendem Gewebe bestehende Auflagerungen. Am Endokard der hinteren Wand der linken Kammer in der Ausbreitung von Kronengröße sind blutig imbibierte Striemen zu sehen. Lungen nicht gebläht, füblen sich wie Schwamm an, die braunrote Schnittfläche ist mit seröser, blutiger, schäumender Flüssigkeit durchtränkt. In den Bronchien viel zäher Schleim. Schleimhaut der Bronchien blutreich. Peribronchiale Lymphdrüsen klein, weich, schwarz. Aortenbogen glatt. Die Schleimhaut der Luftröhre, Speiseröhre, des Rachens, Kehlkopfes etwas blutreich. Schilddrüse mittelgroß.

Die Kopfhaut ist mitteldick, mittelblutreich. Schädeldach mitteldick, mit entsprechender Diplöe. $178 \times 150 \mathrm{~mm}$. Die harte Gehirnhaut läßt sich leicht vom Gehirn abziehen, in den Blutleitern viel flüssiges, dunkelrotes Blut. Die weiche Gehirnhaut dünn, etwas blutreich und ödematös durchtränkt. Gehirnarterien dünnwandig. Das Gehirn wurde in frontale Schnitte zerlegt. In der Höhe der Zentralwindungen, in den beiden Marklagern fand sich je ein über bohnengroßer, etwas eingezogener Herd, dessen Umgebung mit einem kaum sichtbaren, hellen, rosafarbenen Ring umgeben war, dieser Herd beginnt in der Höhe der dritten Frontalwindung und reicht nach hinten im Marklager bis zur hinteren Zentralwindung.

Die Seitenventrikel sind etwas erweitert, enthalten massig viel, etwas leicht getrübten Liquor. Im Thalamus beiderseits 3-4 kleine, nadelstichgroße, $1-2$ steck- 
nadelkopfgroße, scharfumschriebene Herdchen, diese Herdchen wie die großen Herde sind symmetrisch im Marklager, oberhalb des Thalamus gelegen. Dieselben sind leicht unter die Schnittfläche eingesunken, häufig haben sie ein punktförmiges, dunkelgefärbtes Zentrum. Der Herd unterscheidet sich von der Umgebung und ist etwas haloniert. Der Thalamus und die Gehirnschenkel mit dem Kleinhirn und Brücke wurden sorgfältigst untersucht, es fand sich aber keine makroskopische Veränderung.

Diagnose: Pyämie, Endocarditis valvulae bicuspidalis. Degeneratio parenchymatosa myocardii, adiposa renum (Chorea minor), Emolitio Substantiae albae cerebri magnitudinis nucis juglandis, lat. utriusque.

Ein Teil der Frontalschnitte des Gehirns in der Höhe der beiden Thalami wurde in Mülller - For mol gelegt und nach Weigert auf Markscheiden untersucht, ein Teil wurde in Alkohol nach Nissls Methode, Gefrierschnitte wurden nach Bielschowsky, nach Best, nach Herxheimer, Alzheimer, Mallory und nach verschiedenen anderen Methoden und auch nach Ramony-Cajal gefärbt und untersucht.

Im Subarachnoidalraum des Gehirnes sind an vielen Stellen starke, große Erythrocytenansammlungen anzutreffen, auch an den tieferen Stellen der Furchen sind Anhäufungen von Leukocyten. Hier und dort findet man thrombosierte Gefäßchen der weichen Gehirnhaut um die Lumina der Gefäßchen, von einem Ring von mehrkernigen Leukocyten und von Kokkenhaufen umgeben. Die Arterien zeigen eine starke Füllung mit roten Blutkörperchen. Auffallend ist eine die motorische Bahn begleitende gewerbliche Veränderung, die völlige Ausfüllung der Capillaren des Marklagers und der inneren Kapsel, dann des Thalamus beiderseits, beider Bindearme, sowie der roten Kerne und Nucleus dentatus, Fasergebietes mit Kokkenpfröpfen, die aus dicht nebeneinandergepreßten, zu einem Stöpsel zusammengedrückten Kokkenmassen bestehen. Solche Kokken mit Leukocytenkörnchen gemischte Pfröpfchen sind im Verlauf der ganzen motorischen Bahn fast überall, fast in jedem Gesichtsfelde zu sehen, hingegen finden sich solche außer der motorischen Bahn überhaupt nicht. Dieses Verhalten kann manan den großen Hemis phärenparaffinschnitten, die ich in Chloroform - Paraffineinbettung ganzer Hemisphärenstücke und Schneiden der Riesenblöcke unter Wasser nach der Methodevon Prof Dr. Orsos herstellte, leicht und bequem studieren. Es hatte sich bei der mikroskopischen Untersuchung ergeben, daß viel mehr miliare Herde und Herdchen im Gehirn, im Marklager anzutreffen sind, als man geglaubt hatte. Besonders im Thalamus und der Gehirnrinde sind viele Herdchen. Miliare Ansammlungen von Eiterzellen sind in der Umgebung der im Marklager liegenden zwei größeren Herde, die noch mit Kokkenmassen vollgefüllt sind. Die Gefäßwand kann an der Stelle der Embolisierung nicht immer gut nachgewiesen werden, nicht einmal die elastischen Fasern, der Pfropf war ja größer als das Gefäßchen, es war also an vielen Stellen Nekrose der Gefäßßwand zu sehen; die Bakterien überwucherten die nekrotisierte Gefäßwand an vielen Stellen. Um den Embolus herum finden wir an vielen Bezirken polymorphe und mehrkernige Leukocyten.

Wenn ich nun die Schilderung der Befunde und der mikroskopischen Bilder unterbreche und auf die Krankengeschichte der übrigen Fälle übergehe, so geschieht dies aus Ökonomie, da ich vieles mitteilen müßte, was Nauwerk, Reichardt, Dana usw. beschrieben und in ihren Arbeiten veröffentlicht haben. Ich werde also, nachdem ich die Kranken- 
geschichten des II., III. und IV. Falles mitgeteilt habe, auf die gemein same Schilderung der histologischen Befunde der in klinischer, wie anatomischer Hinsicht in manchen Punkten so verschiedenen, in den gewichtigsten, prinzipiellen Punkten aber ganz gleichen geweblicher Veränderungen eingehen. Es wird sich zeigen, wie verschiedene Symptome und gewebliche Veränderungen der Staphylococcus pyogenes aureus verursachen kann, doch werden sich aus der Untersuchung der vier Gehirne, speziell aus der feineren Histologie der vier anscheinlich verschiedenartig lädierten Gehirne, allgemeinere, auf alle vier Gehirne gültige Gesichtspunkte gewinnen lassen.

Der zweite Fall betrifft ein 17 Jahre altes Mädchen, die am 26. April 1916 in die psychiatrisch-neurologische. Universitätsklinik aufgenommen wurde und am 4. Mai 1916 an schwerer Chorea infectiosa, Erschöpfung und Erscheinungen der Sepsis starb.

Anamnese: Seit einer Woche krank, aber arbeitete noch in einer Fabrik. Seit einigen Tagen ziemlich heftige, andauernde choreatische Bewegungen, besonders der oberen und unteren Extremitäten. Appetit ist gut, schlaflos.

Status praesens: Massig entwickelte, schwach ernährte Kranke, mit enger Stirn. Iris blau, Pupillen reagieren auf Licht und Akkomodation gut; Zunge belegt, Lippen trocken, Temperatur $38,2^{\circ}$, Puls $112-120$, Herzdämpfung etwas nach links verbreitert, über der zweiteiligen Klappe ist ein leises, blasendes, systolisches Geräusch an Stelle des zweiten Tones zu hören. Oberhalb der Lungen perkutorisch keine Veränderung, aber überall, besonders oberhalb beider Unterlappen, hinten, bronchiales, raues Atmen. Die Reflexe sind alle gesteigert. Im Gesicht rege, andauernde Bewegungen. Im Bett haut die Kranke mit beiden Armen herum, wirft die Füße herum, kann keine Minute in Ruhe liegen, äußerst heftige choreatische Bewegungen. An beiden Flanken, an der Kreuzbeingegend, helle große Hautabschlürfungen.

28. IV. 1916. Aus der Vena mediana des rechten Armes wird $10 \mathrm{ccm}$ Blut steril entnommen und in 6 Röhrchen Serumbouillon zerteilt, in denen in 24 Stunden bei $37^{\circ} \mathrm{C}$ eine feine Trübung entstanden ist. Auf Agarplatten wachsen die gelben Kolonien des Staphylococeus pyogenes aureus in Reinkultur aus. Die Herztöne sind rein. Puls 120, weich und klein (Coffein, Campher, Digalen).

Pat. starb am 3. III. 1916. Pat. war also insgesamt 17 Tage krank, lag davon 9 Tage in der Klinik.

Pathologisch-anatomische Diagnose: Endocarditis valvulae bicuspidalis. Intumescentia lienis mediocris gradus, degeneratio adiposa cordis, renum, hepatisque. Hypertrophia tonsillarum (Chorea infectiosa) Sepsis. Decubitus incipiens.

Fall 3. Frau M. M., 22 Jahre alt, wurde von der 2. Internen Klinik wegen äußerster motorischer Unruhe am 24. X. 1918 in die psychiatrisch-neurologische Universitätsklinik gebracht.

Anamnese: Hat ein gesundes Kind, welches Pat. 13 Monate stillte; nachher bemerkte sie, daß im linken Arm Zuckungen auftraten und der linke Arm stark zittert. Wurde auf der Ambulanz der Klinik behandelt und geheilt. Seit September gravid, mit der auch die Zuckungen wiederum beginnen, aber mit viel größerer Intensität wie vor einem Jahr. Am 14. X. schleppte Pat. einen schweren Korb nach Hause, und als sie die Last niederlegte, steigerte sich die Heftigkeit 
der Zuckungen von Tag zu Tag, Pat. wurde immer unruhiger. Pat. konnte selbst nicht essen, fiel vom Bett, hatte sich stark angeschlagen. Sprache unverständlich, völlig unartikuliert. Temperatur $38,2-38,5^{\circ}$, Puls 120, weich, arythmisch (Campher, Digalen). Am 26. X. 1918. Die Jaktationen äußerster Heftigkeit dauern bis heute früh, aber Zuckungen sind noch in den Gesichtsmuskeln vorhanden, die Augäpfel rollen von rechts nach links. Um $10 \mathrm{Uhr}$ vormittags werden zwei $5 \mathrm{~cm}$ lange Föten geboren, die Placenta stößt sich von selbst ab. Temperatur $39^{\circ}$, Puls 125.

Am 27. X. 1918. Puls weich, arythmisch, 120. Temperatur $38,9^{\circ}$. Die Kranke kann nur ja und nein sagen. Das Schlucken ist sehr erschwert, soporös. Es sind noch vereinzelte choreatische Zuckungen in den Armen, Beinen zu sehen, in den Gesichtsmuskeln choreatische Bewegungen, Zunge wird oft herausgestreckt. Der Puls wird immer schwächer und ist am Abend kaum tastbar und Pat. stirbt an Herzschwäche um 8 Uhr abends.

Noch am 25. X. werden aus der Vena mediana $10 \mathrm{ccm}$ Blut steril entnommen und auf Serumagarplatten verarbeitet, auf denen während $2 \times 24$ Stunden der Staphylococcus pyogenes aureus auswächst.

In diesem Falle haben wir es mit einer 22 Jahre alten Frau zu tun, die ins. gesamt 57 Tage krank war und 3 Tage in unserer Klinik gelegen hat. Die erste Erkrankung, die 30 Tage dauerte, war vor $1 \mathrm{Jahr}$ gewesen.

Qbduktions protokoll: Die Kopfhaut ist mitteldick, mittelblutreich, das Schädeldach mitteldick, rund, mit entsprechender Diplöe. Die harte Gehirnhaut ist im allgemeinen weißlich verdickt, mittelblutreich. Das Gehirn ist mittelgroß, die weichen Gehirnhäute sind etwas trüb und ödematös. Die Win. dungen des Gehirns sind gut entwickelt. Oberhalb der rechten Hemisphäre in der Gegend der hinteren Zentralwindung ist unterhalb den weichen Gehirnhäuten eine kinderhandtellergroße Blutung, das Blut ist in einer $0,2 \mathrm{~mm}$ Dicke ausgebreitet, entsprechend dem oberen Viertel der rechten Zentralwindung befindet sich eine nußgroße Höhle. Die Wand der ziemlich großen Höhle ist glatt, an einigen Stellen aber franzig, von etwas grauer Farbe, die Höhle selbst ist mit flüssigen und etwas halbgeronnenem Blut ausgefüllt, welches aber nirgends an die Wand des Herdes anhaftet. Im rechten Gyrus centralis anterior und in den rechten und linken Stirnwindungen sind immens viele mittelgroße, feinste Capillaren mit hellroten Thromben verstopft, auch sind 5-6-8 subcorticale Gefäßchen mit ganz frischen, hellroten Thromben versehen. Das Gewebe um die corticalen und unterhalb der Rinde gelegenen Gefäßverstopfungen sieht makroskopisch unverändert aus, es sind keine Erweichungen, keine Hallonierung, und keine Verfärbung des umgebenden Rindengewebes noch des subcorticalen Marklagers zu beobachten. Die Seitenventrikel sind mittelwert, das Ependym ist glatt. Das Gehirn ist überall blutreich, seine Konsistenz ist mittelfest.

Diagnose: Polioencephalitis haemorrhagica haemisphaerae cerebri dextrae cum ruptura arteriae convexitatis cerebri dextri sub gyr. centr. posterioris dextri. Emolitio substantiae gyri central. posterioris dextri magnitudinis nucis avellanae cum haematoma ibidem magnitudinis nucis avellane. Thrombi arteriarum gyri centralium ant. et gyri front. III. lat. dextri et sinistri recentes. Endocarditis verucosa valvulae mitralis. Intumescentia lienis minimi gradus infaretus quatuor lienis haemorrhagici. Degeneratio parenchymatosa cordis, renum, hepatisque maioris gradus. Sepsis. Anaemia universalis mediocris gradus. Chorea infectiosa. 
Fall 4. I. K., Mädehen, 16 Jahre alt, wurde am 31. VIII. 1918 in die psychiatrisch-neurologische Universitätsklinik aufgenommen.

Mitte Februar 1917. bemerkt man, daß in dem rechten und linken Arm, und im rechten Bein der Kranken unwillkürliche Zuckungen sich einstellten, diese Zuckungen wurden immer heftiger, so da $B$ Pat. nicht gehen und nicht essen konnte. Pat. wurde in das Kinderspital gebracht, wo sie wegen Chorea infectiosa behandelt wurde, es hörten die Zuckungen auf, aber es haben sich gewisse Lähmungserscheinungen eingestellt. Die Kranke konnte ihre Arme nicht bewegen, sie konnte mit ihren Händen nichts erfassen, so daß man sie Füttern mußte, auch konnte sic nicht stehen. Klagte über immens heftige Schmerzen in den Beinen.

Am 28. XI. 1918. Temp. 40. Starke Kopfschmerzen. Herzarbeit zeitweise unregelmäßig, auslassend. Pat. bekam die spanische Grippe. (Campher und Digalen.) Über beide Lungenunterlappen ist der Percussionsschall etwas gedämpft, das Atmen rauh, bronchial, überall Giemen, Schnurren, Pfeifen. In den Armen, Händen, Schultern und Gesichtsmuskeln lebhafte choreatische Bewegungen.

Am 30. XI. 1918. Die Herzaktion ist zeitweise auslassend. Starkes Nasenbluten. Über den beiden Lungenunterlappen verkürzter Percussionsschall, Rasselgeräusche, Giemen, und Pfeifen, rauhes bronchiales Atmen über beide Lungenoberlappen. Temp. 38,9.

Am 1. XII. 1918. Lebhafte choreatische Zuckungen der oberen Extremitäten, in den unteren zeitweilige tonische Spannungen der Muskulatur.

Am 2. XII. 1918 Temp. 39. Phls weich, auslassend, irregulär, über dem rechten Unterlappen völlige Dämpfung, nasse Rasselgeränsche. Pat. ist sehr verfallen, die choreatischen Zuckungen und die inkoordinierten Bewegungen in den Gliedmaßen dauern fort.

Am 4. XII. 1918 Temp. 39. Puls filiform, irregulär. Oberhalb der linken Lungenunterlappen völlige Dämpfung, bronchiales Atmen. Um 4 Uhr nachmittags stirbt Pat. an Herzschwäche infolge der beiderseitigen Lungenentzündung.

Obduktionskrotokoll: I. K., 16 Jahre alt. Die Aortenklappen sind membranös, die zweiteilige Klappe zurückgezogen an den Rändern mit 4-6 mm dicken, knotigen, durchsichtigen Belag, von da etwas nach innen zu sind weißliche, strahlige Narben an den Klappen vorhanden. Die Seiten sind verkürzt, verdickt, die Trabekeln sind abgeflacht, die Coronararterien sind ohne Befund. Das viscerale Perikard ist glatt, glänzend. Beide Lungen kollabieren nicht, das Brustfell über beide Lungen ist glatt, glänzend, nur an den Rändern ist ein feiner, fibrinöser Belag sichtbar. Die rechte Lunge ist an der Basis und hinten und unten mit fibrösen Bändern mit dem Brustkasten und Zwerchfell verwachsen. Die Lungenschnittfläche ist intensiv dunkelrot, beide Unterlappen und ein Teil der Oberlappen hat fast eine leberartige Konsistenz. Die Schnittfläche an diesen eben erwähnten Stellen ist mappenartig, fleckenweise wechseln sich dunkelschwarzrote und rote Flecke ab. An der dunkelroten geschwollenen Bronchialschleimhaut viel zäher, festhaftender Schleim. Die Schleimhaut der Luftröhre ist sehr blutreich, speziell an der Stelle der Zweiteilung ist dieselbe sehr verändert, mit grauweißem, strähnenartigem Belag bedeckt. Die Milz ist $13 \mathrm{~cm}$ lang, die Ränder dick, die Kapsel angespannt, die Substanz der Milz ist schlaff, die Schnittfläche sehr blutreich, stellenweise mit Blutungen in das interstitielle Gewebe. Die Zeichnung und die Follikeln gut sichtbar.

Gehirn. Die Dura ist blutreich, mitteldick, im langen Blutleiter massig viel rotes, flüssiges Blut. Dünne Gehirnhäute sind blutreich, etwas ödematös durchtränkt. Die Seitenventrikel sind etwas erweitert, die Gehirnrinde und das Mark deutlich blutreich, besonders die Thela chorioidea. Die Substanz und das Gehirn weich, nur in dem Thalami etwas kompakter. Thrombosierungen, Embolien, Erweichungen sind weder im Großhirn noch im Kleinhirn nachweisbar gewesen. 
Diagnose: Pneumonia catarrhalis partim haemorrhagica loborum inf. totius pulmonum utriusque et partim lobor. superiorum. Bronchitis et tracheitis fibrinosa acuta. Pleuritis fibrinoso-fibrosa lateris dextri. Tumor lienis acutus. Degeneratio parenchymatosa cordis, adiposa hepatis et renum. Haematoma parietis abdominis musculorum rectorum abdominis magnitudinis pugni virilis. Endocarditis verrucosa et retrahens verosimiliter rheumatica, valvulae bicuspidalis. Cholecystitis chronica fibrosa. Hydrocephalus chronicus mediocris gradus internus, Hyperaemia cerebri mediocris gradus. Febris hyspanica. Chorea minor.

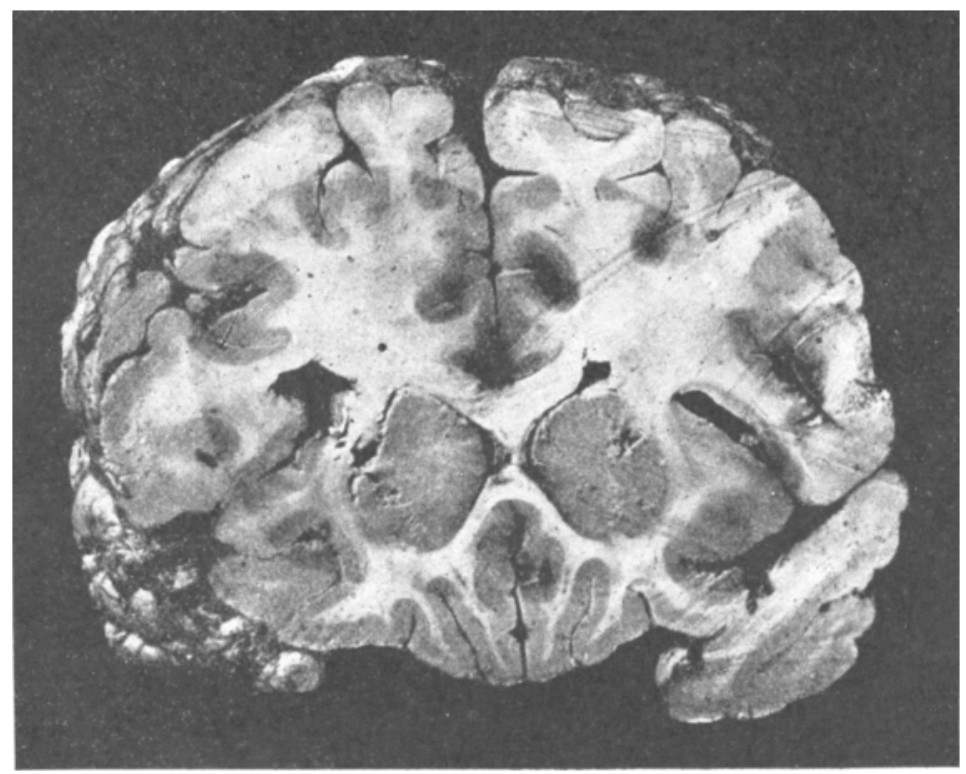

Abb. 1. 'Gehirn von Fall 2. Beginn der Höhlenbildung und Erweichung der äußeren Kapsel.1)

Die mitgeteilten Krankheitsfälle haben viel Gemeinschaftliches, indem bei allen vier Fällen choreatische Zuckungen bestanden, einmal kompliziert mit einer schweren Neuritis und in einem Falle entstand das Chorearezidiv während der Schwangerschaft. In drei Fällen konnte in vivo der Staph ylococcus p yogenes a ure us a us der Blutbahn rein ge züch tet werden. In allen vier Fällen fand sich bei der Autopsie Endocarditis der Mitralklappen. Bei der mikroskopischen Untersuchung fanden sich in den Capillaren aller vier Fälle, aber nur in den Capillaren der Zentralwindungen der Thalami, der Bindearme und die des roten Kerns mit Kokken so vollgefüllt, daß diese aus den Kokken bestehenden

1) Alle Mikrotophogramme sind mit dem kleinen mikrophotograph. Apparat von Zeiss, hergestellt, mit dem Projektionsokular Nr. 4. Homogenimmersionslinse 1,3. Apertur. Kameraauszug $60 \mathrm{~cm}$. 
Pfröpfe an vielen Stellen die Nekrose der Capillarwände verursachten. Der zweite Fall zeichnet sich durch die im Marklager gelegene unterhalb der dritten rechten Frontalwindung beginnende in der Höhe der rechten Parietooccipitalwindung, nach hinten zu im Durchmesser immer kleiner werdende horizontal gelegene, glattrandige Höhlenbildung (s. Abb. 1, 2,3) aus.

Im dritten Fall hingegen finden wir eine unterhalb der rechten, hinteren Zentralwindung gelegene, mit flüssigen, roten und sehr geringer

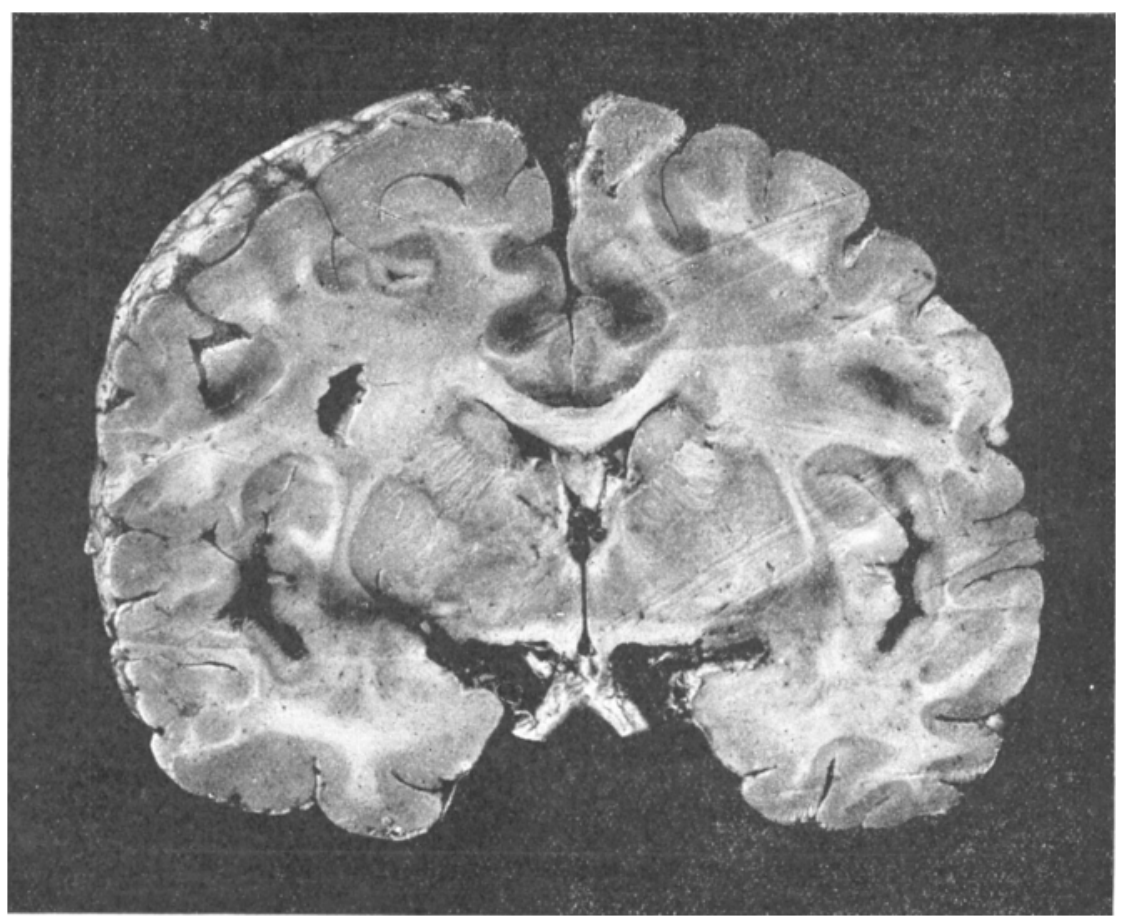

Abb.2. Gehirn von Fall 2. Fortsetzung der Höhle in der rechten Hemisphäre im Marklager.

Menge halbgeronnenen Blutes gefüllte Höhle, verbunden mit einer ganz frischen Polyencephalitis haemorrhagica dextra als Todesursache.

In dem vierten $\mathrm{Fall}$ sieht man hingegen mit freiem Auge außer einen geringen Hydrocephalus und eine geringe Hyperämie der Rinde und des Marklagers keine Veränderungen.

Alle vier Gehirne wurden nach einem Plan aufgearbeitet, in frontaler Richtung in $11 / 2 \mathrm{om}$ dicke Schnitte zerlegt, einige Stücke in Müllerformol gelegt und nachher in Zelloidin geschnitten und die Hemisphärenschnitte nach WeigertWolters, nach Weigert - Pal und Kultschitzky gefärbt. Außer der Nisslmethode, der Tolluidinfärbung, wurde die Silberaldehydmethode von Bielschowsky und alle Gliamethoden, die Fettfärbung von Herxheimer, die die Elasticafärbung von Weigert angewendet. 
Beitrag zur Histopathologie und Bakteriologie der Chorea infectiosa. 341

Bei der Lösung verschiedener Probleme der Pathologie und pathologischen Histologie gewinnt ein gewisses Verfahren eine überwiegende Bedeutung, wenn es auf die gestellte Frage eine Antwort richtig und eindeutig geben kann bzw. in der Lösung der Probleme dem Anwender der Methode einen komplizierteren Proze $ß$ zu verstehen verhilft. Die übrigen Methoden ergänzen, bestätigen die Befunde oder beleuchten den pathologischen Proze $B$ von einer anderen Seite. So tritt die vorzügliche Hämatoxylin-Eosinfärbung vor der Methylenblaufärbung bei der Untersuchung des Zentralnervensystems zurück, und die Gliamethode Weigerts muß durch das Protoplasma der Gliazellen darstellenden Verfahren

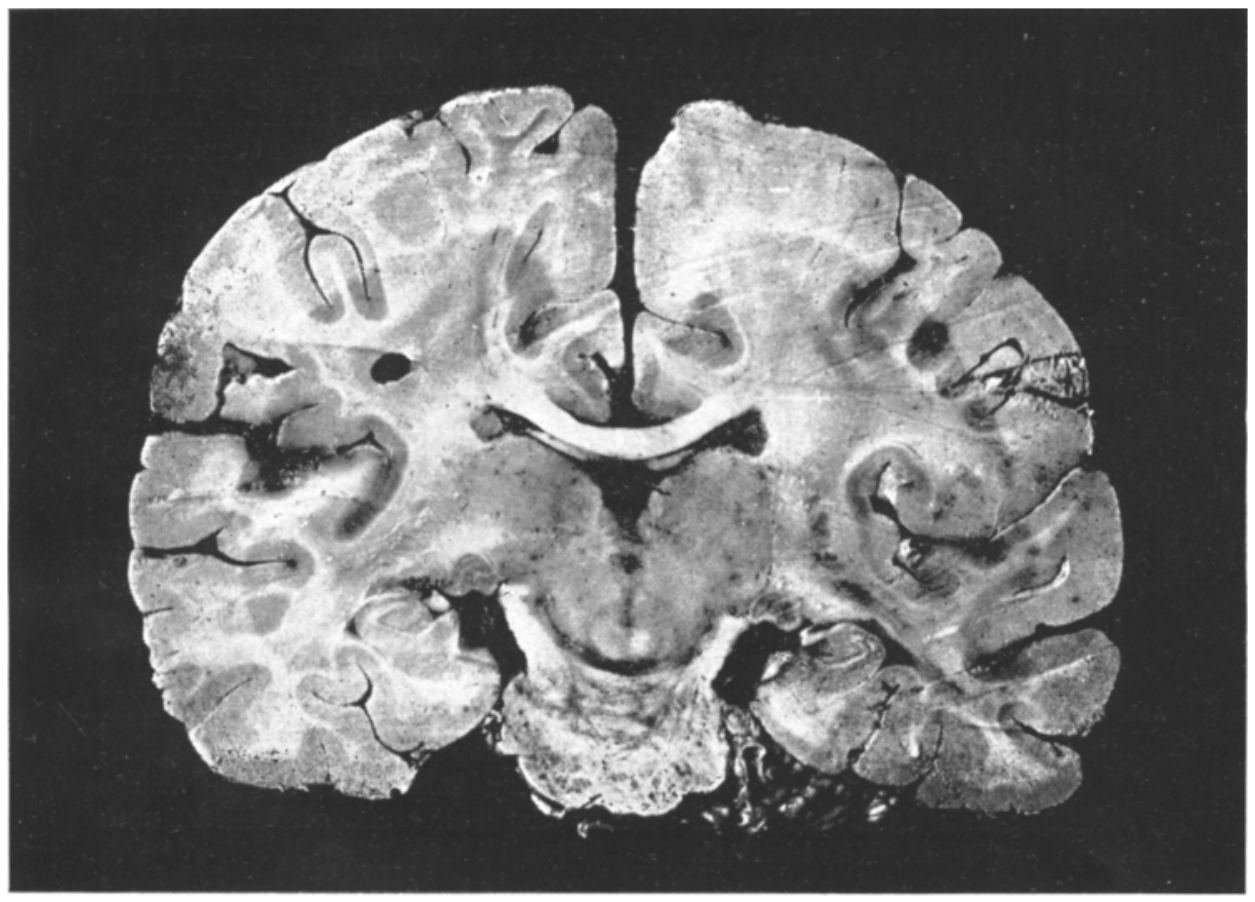

Abb. 3. Ende der Höhle im Marklager vom Falle 2.

von Herxheimer, Mallory, Alzheimer, Mann, von Eisath, Held und der neueren Gliamethode von Ra mon y Cajal ergänzt werden, da doch die klassische Methode Weigerts nur die Kerne und Gliafasern zur Darstellung bringt.

Die Methode Cajals wurde bald von Schaffer auf ihren Wert in normalen Verhältnissen und bei pathologischen Prozessen geprüft, und er bestätigt die Vorzüge der einfachen Handhabung und die Klarheit der Bilder bei pathologischen Prozessen, die von Cajal und dessen Schülern betont wurden.

Man unterscheidet I. Ersatzwucherung (Weigert), bei der es zur Bildung von Spinnenzellen kommt. Der Zelleib schwillt an, an den Kernen kommt es zu Proliferationserscheinungen, dann bemerkt man direkte und indirekte Kernteilung, als Produkt dessen mehrkernige 
Zellen, Zelleibanschwellen, so auch die Fortsätze. Große, immens faserreiche Gliazellen kommen zustande, die aber auch bald schrumpfen, der Kern verändert sich, die Fortsätze werden zerklüftet, die Gliazellen gehen unter. Bielschowsky beschrieb bei der tuberösen und hypertrophischen Hirnsklerose Zellen, welche das Stadium der protoplasmatischen Astrocyten nicht überschreiten und Gliafasern nicht bilden, es sind das Übergänge von fibrösen Astrocyten und Fortsatz und plasmaarmen Gliazellen, dieselben sollen mangelhaft differenzierten Urepithelien ihre Herkunft verdankøn.

Bei der mikroskopischen Untersuchung der vier Gehirne wurden von mir horizontale, große Scheiben aus dem Lobus frontalis, Lobus occipitalis, Lobus parietalis, Stücke aus der Höhe der bei-

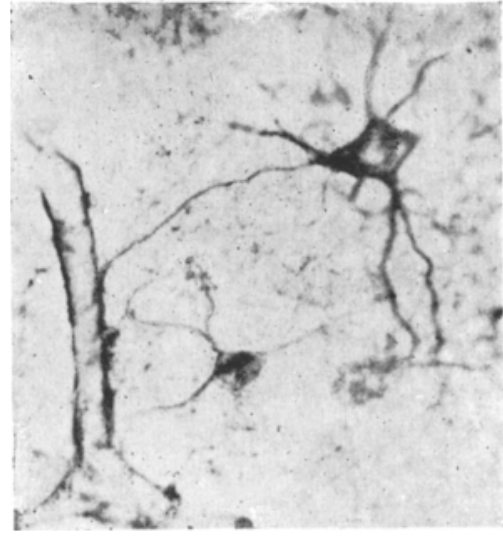

Abb. 4. Normale Gliazelle, mit feinen Gliafiißchen zu einer Capillare ziehend. den Zentralwindungen aus dem Klein hirn, aus dem verlängerten Mark von der Dicke von $3-4 \mathrm{~mm}$ ausgeschnitter, dieselben auf dem nach meiner Angabe durch die Firma Reichert gefertigten großen Gefriermikrotomtisch geschnitten (Schnittdicke $8-10-18 \mu$ ); so kann man sog. Riesengefrierschnitte verfertigen, die $1 / 3$ einer Hemisphäre enthalten, wobei z. B. alle Frontalwindungen und deren Marklager auf einen Schnitt tadellos sichtbar sind oder der Thalamus mit den Windungen der Insel usw, Diese Gefrierschnitte wurden in Gliabeize gelegt, nach Malory, Alzheimer, Mann gefärbt, andere wurden ohne Beize nach Cajal behandelt, wiederum andere Schnitte, nachdem sie in destilliertem Wasser gewaschen wurden, nach Bielschowsky behandelt. Andere Stücke des Gehirns aller Fälle wurden in Paraffin eingebettet und geschnitten, nach Lenhossek, Held, Nissl gefärbt.

Die Ergebnisse, die ich an den großen Gefrierschnitten mit der Ramon y Cajalschen Brom-Formalin-Sublimat-Goldchloridmethode erreicht habe, sind so frappierend klar und eindeutig, daß ich aus Ökonomie mit der Schilderung der Cajalbilder beginne, die Bilder der mit den übrigen Gliafarbeverfahren anfügend beschreiben werde, da sie ergänzend wirken werden. Außerdem fand sich aus diesen Cajalbildern der Gewinn, daß ich über die Befunde, da sie völlig übereinstimmend sind bei allen vier Gehirnen, gleichzeitig gerichten kann.

Auffallend war, daß in der Gegend und Umgebung der großen Läsionen, der Höhlenbildung im dritten Falle, in der Umgebung der mit frischem Blut gefüllten, großen Höhle, unter der rechten Zentralwindung überhaupt keine Gliareizreaktion, wie Vergrößerung der protoplasmatischen Gliazellen, Vermehrung der protoplasmatischen Glia- 
zellen zu finden waren, weder Nekrose und Gliatod, wie sie von Cajal und von Schaffer bei der Paralyse und bei der Idiotie und Epilepsie speziell aber eben bei der Paralyse gefunden und beschrieben hatte. Wir finden überall die normalen Typen, wie sie Cajal und Schaffer abbilden, Abb. 4 zeigt eine normale Gliazelle der Rinde. Im Verlauf der motorischen Bahn, von den Zentralwindungen beginnend bis $\mathrm{zu}$

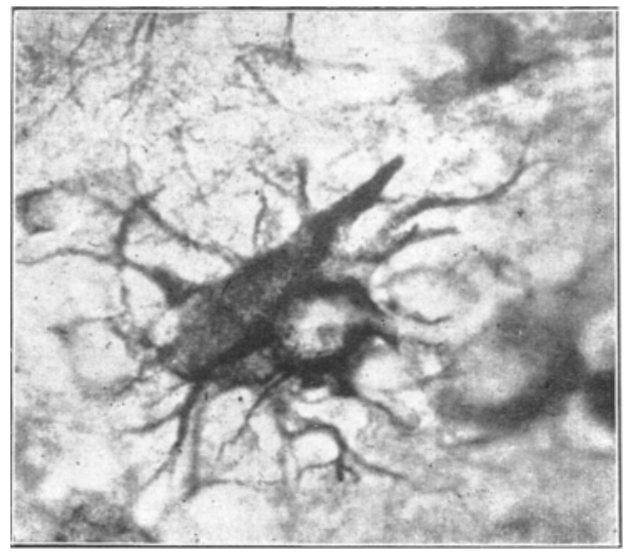

Abb. 5. Nekrotisierende, hypertrophische, protoplasmatische Gliazelle der Zentralwindung. den Bindearmen und bis zu den Nuclei dentati der Kleinhirne unterscheiden sich die Gliafasern und protoplasmatischen Gliazellen scharf von den übrigen Bahnen des

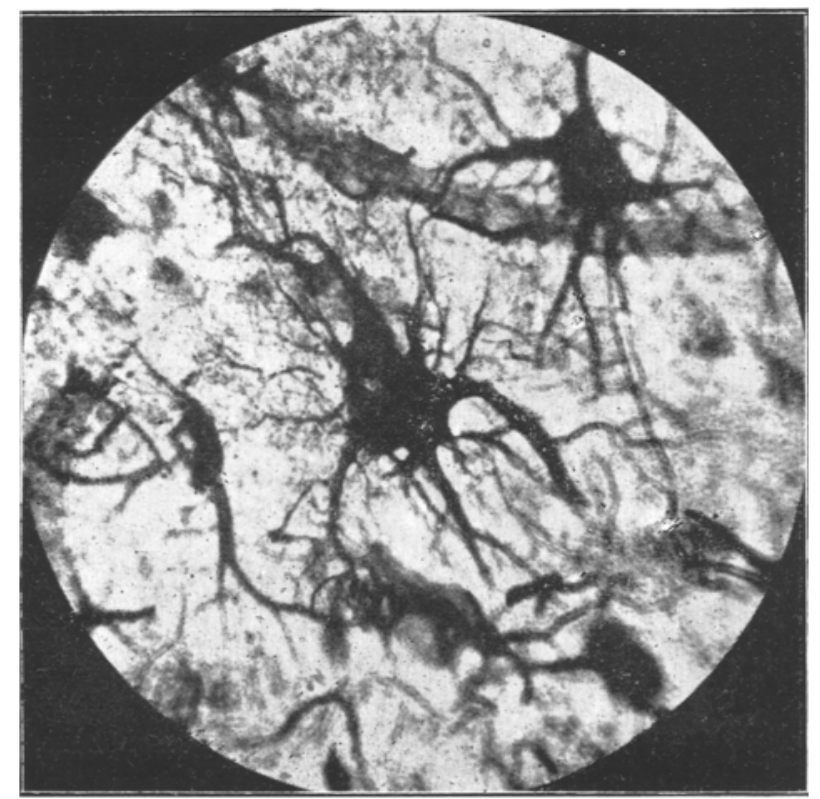

Abb. 6. Hypertrophische, dickfaserige, protoplasmatische Gliazelle der Zentralwindung.

Gehirns dadurch, daß die Gliazellen vergrößert, außerordentlich hypertrophisch sind, außerdem sind die protoplasmatischen Gliazellen ver- 


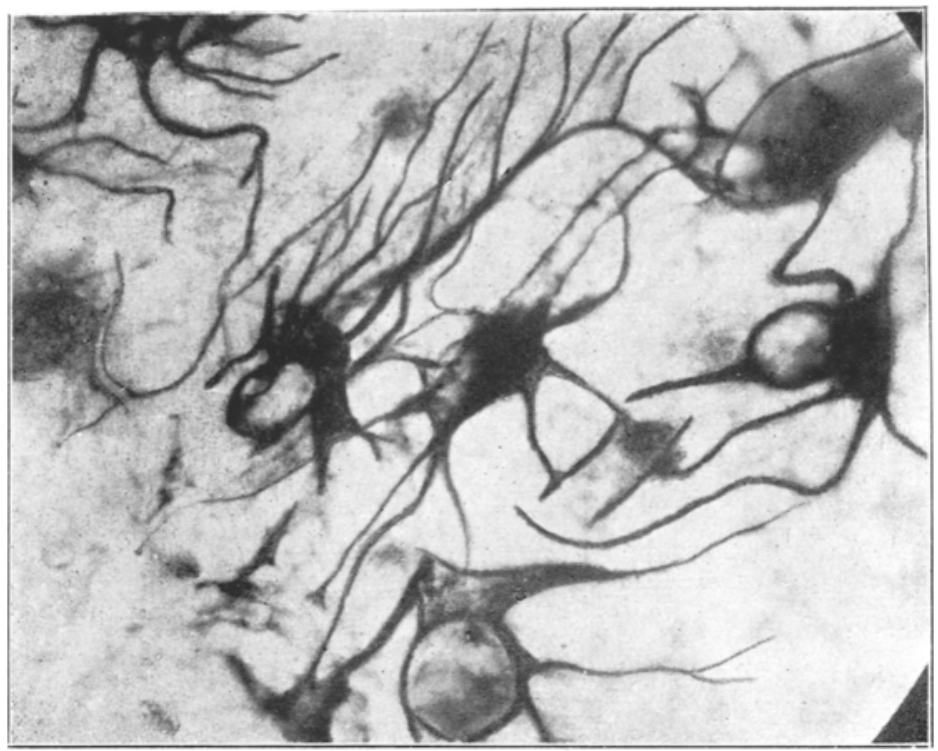

Abb. 7. Hypertrophisehe Gliazellen im Gyr. antr. zu Ganglienzellen ziehend.

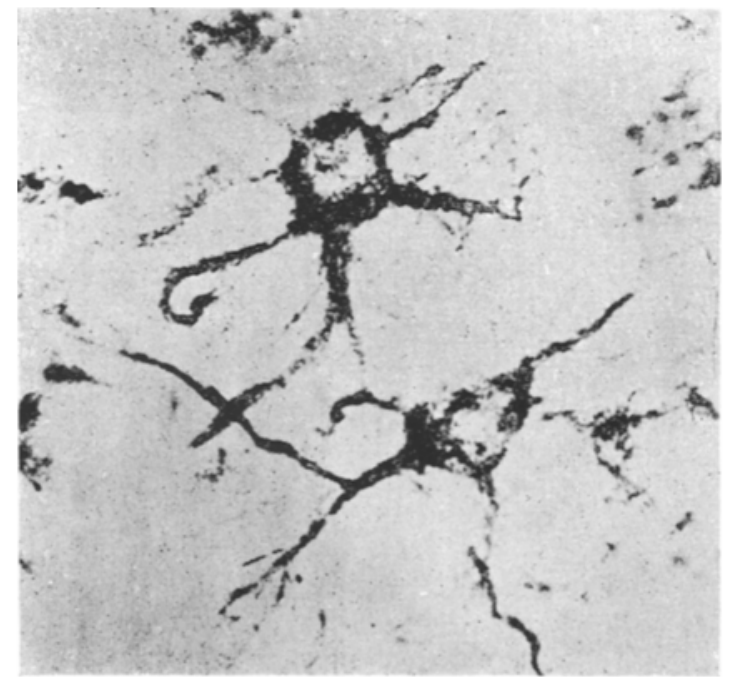

Abb. 8. Schwere Glianekrose im Gyr. centr.: protopiasmatische Gljazellenschatton. mehrt, stellenweise schon einem schweren, unverkennbaren Untergang unterliegend und die verschiedensten Stadien der Glianekrose zeigend (Abb. 5, 6, 7, 8). Im Mark hingegen ist die Verfaserung der Gliazellen sehr ausgesprochen.

In der zonalen Sehicht der Zentralwindungen sind die Gliazellen Cajals vergrößert, zu den feinen Capillaren zichen dieselben mit breiten Gliafasern und enden daselbst mit deutlich stark verbreiterten Gliaendfüßchen (Abb. 8, 9). In allen vier Fällen, die ich untersuchte, habe ich die- 
selben, eben jetzt beschriebenen Befunde gesehen. In den Schichten der Betzschen Zellen und in der Pyramidenschicht finden sich riesig große Gliazellen, mit großem Protoplasmaleib, mit großen Kernen (siehe Abb. 11), dunklen, dichtliegenden Gliosomen, doch färben sich die Gliazellen so intensiv mit Sublimatgold, daß sie oft nur homogenschwarz gefärbt erscheinen. Man begegnet in diesen Schichten Gliazellen mit baumastähnlichen dicken, gewellt verlaufenden, langen Gliafasern, die übrigen Gliafasern sind atrophisch, abgebrochen, viele zerstäubt (siehe Abb. 10), dann hinwiederum mit protoplasmatischen, zahlreiche mittel-

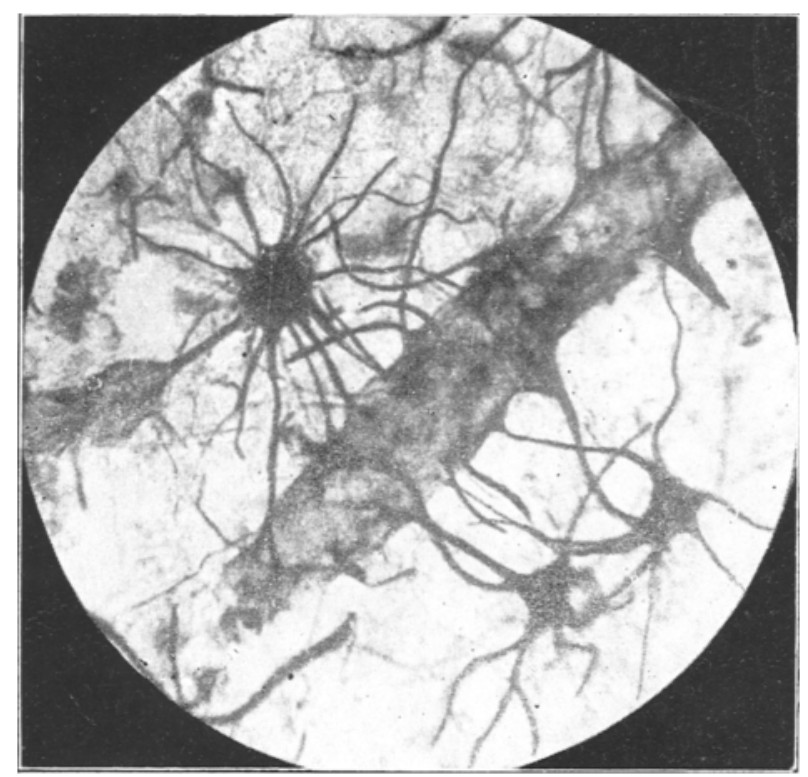

Abb. 9. Gliazellen, vergrößert, mit breiten Gliafüßchen die Membrana perivasc. gliae bilde nd.

dicke, breite Fasern aussendende Gliazellen, die mit breiten Gliafüßen, die sich mit Goldsublimat dunkelviolett färben, an die Capillaren der Rinde enden (Abb. 7, 9). Die schwersten Glianekrosenbilder sah ich in der Rinde in den Zentralwindungen, den Stellen der oberen und unteren Gliedmaßen, weniger ausgeprägt jedoch starke Hypertrophie aufweisend in der Zentralwindung überall. Im Marklager, wo die hypertrophischen Gliazellen mit langen, starken Fasern verlaufen, ist der Unterschied an der Grenze der motorischen Bahn, zwischen umgrenzendem Gewebe und motorischem Gebiet so scharf, wie ich es kaum erwartet habe. Es sind Capillargefäße mit auffallend zahlreichen, vergrößerten, protoplasmatischen Gliazellen und dessen noch zahlreicheren Fasern umgeben, sind ein wahres Geflecht von breiten, dunkelviolettgefärbten 


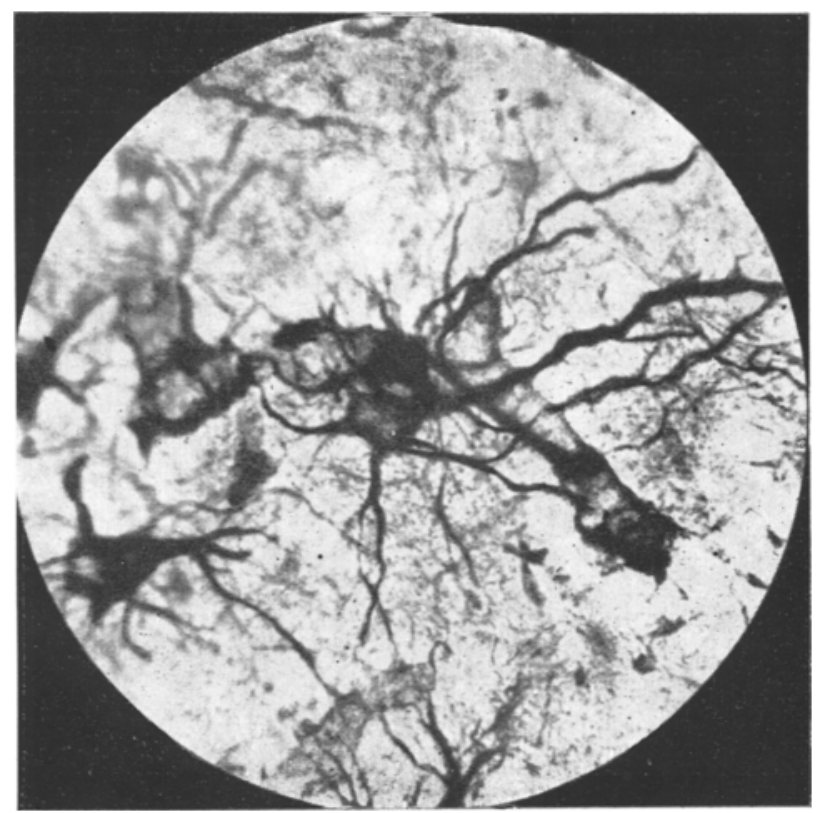

Abb. 10. Gliazelle auz der Zentralwindung, baumastartige, dicke Faser, die meisten Ausläufer sind gebrochen, zerstäubt. Hypertrophie und Nekrose.

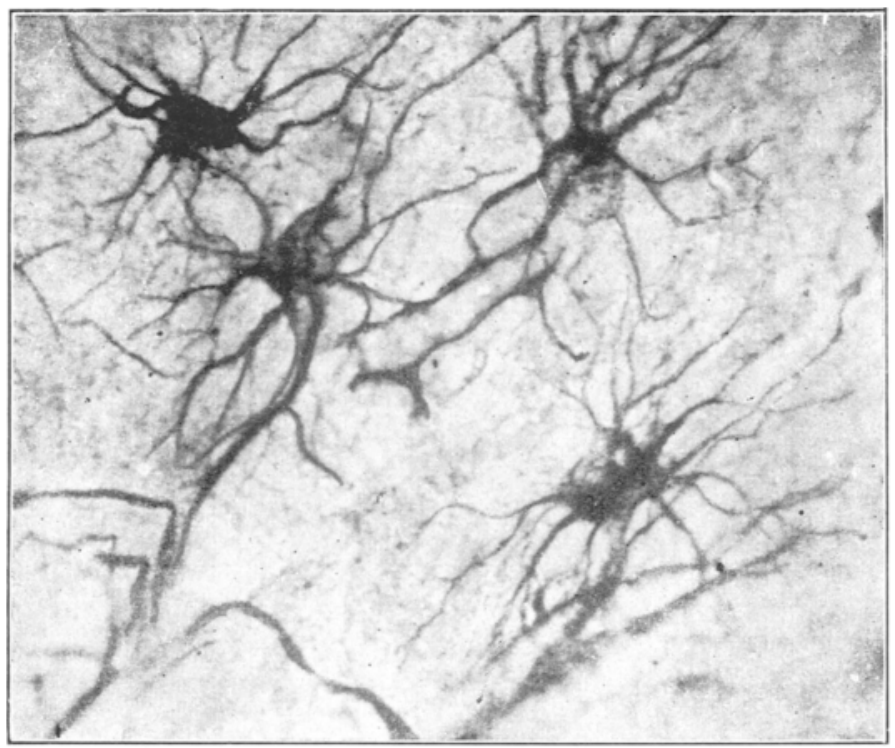

Abb. 11. Quadriga; protoplasmatische, hypertrophische Gliazellen der Zentralwindung. 
Fasern, stark dunkelviolettgefärbten riesenhaften Gliazellen, mit vielen Gliosomen im Protoplasma, die auch dunkelgefärbt sind, vollgepfropft aussehen. Sogenannte ,Quadriga" finden sich auch ziemlich oft, so in der Rinde, wie im Mark (Abb. 11). Ziemlich vergrößerte, dickfaserige, protoplasmatische Gliazellen sind im Nucleus caudatus und Nucleues lentiformis, obzwar die Gliazellen dieser Bezirke in normalen Verhältnissen auch größer sind, wie die der Rinde und noch mehr wie die des Markes (Abb. 12). Die Hypertrophie und der Gliatod ist in den untern Gebieten (Bindearme, roter Kern, Nucleus dentatus) erkennbar, aber nicht so sehr ausgeprägt, wie in der Zentralwindung und im Mark

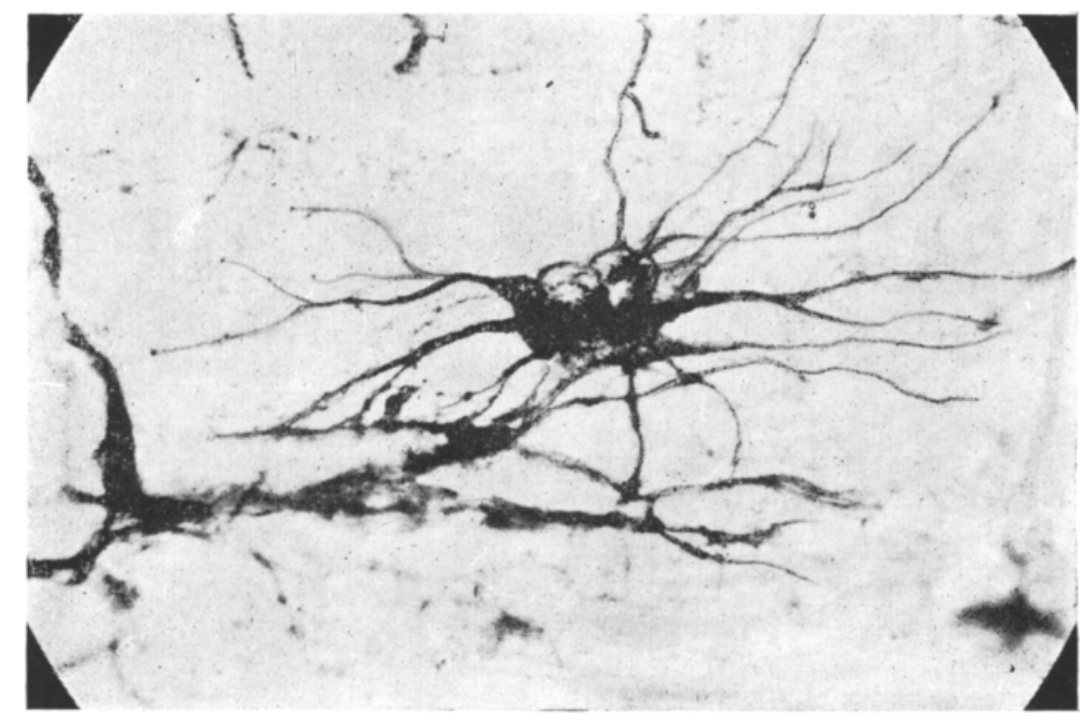

Abb, 12. Mehrkernige, vergrößerte, protoplasmatische Gliazelle mit breitem Gliafüßchen zu einer Capillare ziehend.

(Abb. 13). Wie ersichtlich, vermitteln die Gliazellen eine rege Verbindung zwischen Ganglienzellen und Gefäßen, indem sie mit einem Ausläufer eine Ganglienzelle umfassen, mit einer oder mehreren Fasern hinwiederum ziehen sie zu den Wänden der Capillaren (Abb. 7). So vollführen die Gliazellen eine wichtige biologische Aufgabe in gesunden und krankhaften Verhältnissen, indem sie die verschiedenen Säfte, teils von den Gefäßen zu den Ganglienzellen, von den Ganglienzellen zu den Gefäßen führen. Unzählige Stellen meiner Präparate bestätigen diese Annahme, die Cajal und Schaffer in ihren Werken und Arbeiten ausgesprochen haben. Die Weigertfasern sind im Verlaufe der motorischen Bahnen stark vermehrt, wiederum an andern Stellen der vier Gehirne nicht besonders über die Norm zahlreich vorhanden. Wir 


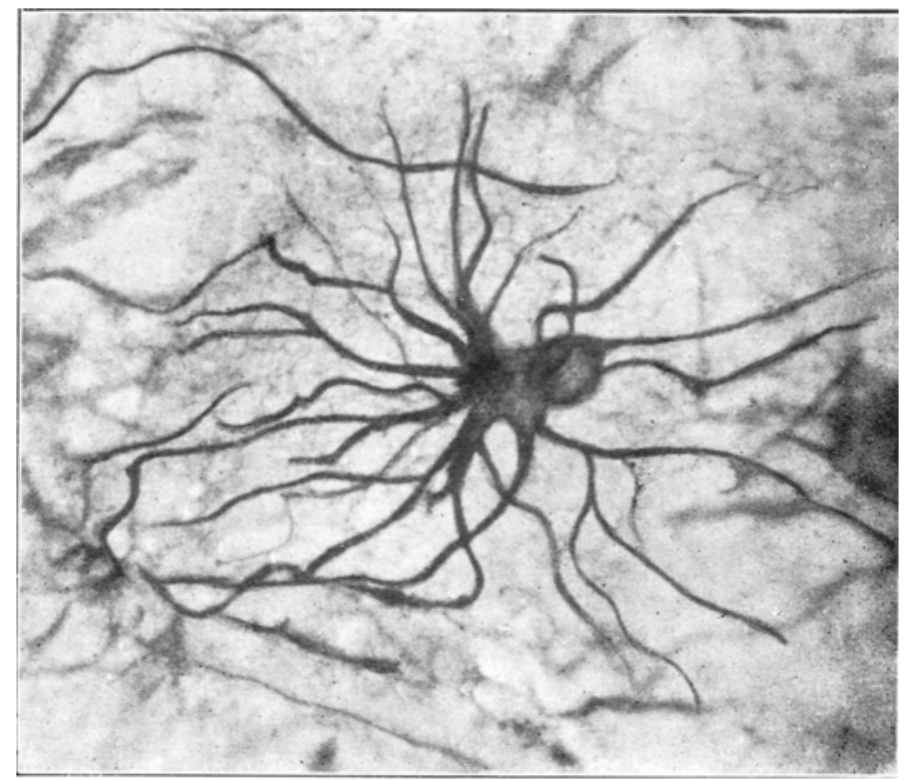

Abb. 13. Hypertrophische, protoplasmatische Gliazelle der Zentralwindung.

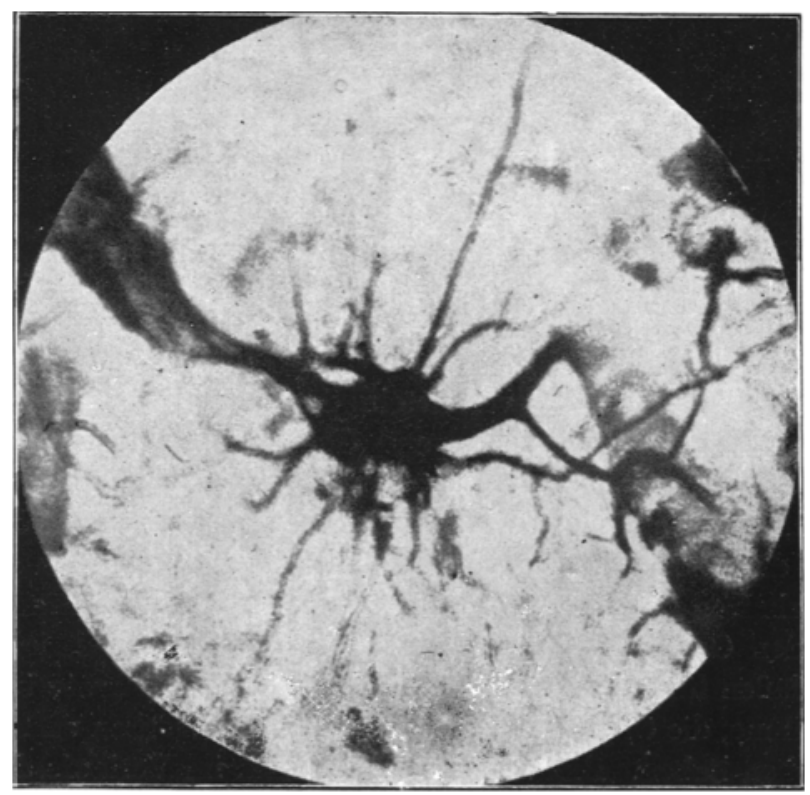

Abb. 14. Nekrotische, protoplasmatische Gliazelle des Nucl, caudatus mit zwei dicken, breites Fortsätzen zu zwei Capillaren hinziehend, die übrigen Fasern sind nekrotisch verdickt. 
stehen also in allen vier Fällen mit einer speziellen, lokalisierten Entzündung der motorischen Bahnen gegenüber, die frischen makroskopisch sichtbaren Veränderungen aber entstanden in der allerletzten Zeit der septischen Erkrankung und hängen mit der Verschlimmerung des Krankheitsprozesses zusammen, bilden nur eine Komplikation der septisch infektiösen Erkrankung.

Die choreatischen Bewegungen würden folglich durch die feste Bindung zwischen den Receptoren der Eiweißmoleküle der motorischen Zellen und der Toxine erklärbar sein, indem durch die Bindung der Toxine in den motorischen Zellen ein Reiz entsteht. Die Hypertrophie der Gliazellen ist der Ausdruck der Reizreaktion der Ganglienzellen, die dieselben durch die Toxine speziell der Bakterientoxine der Choreaerreger, in unseren Fällen also dem Staphylococcus pyogenes albus erfahren. Trotzdem die vier Krankheitsfälle in vielen Beziehungen, speziell in klinischer Beziehung, aber auch in anatomischer Hinsicht, viele verschiedene Verhältnisse aufweisen, sind die feineren anatomischen Veränderungen doch in allen vier Fällen die gleichen. Die feineren histologischen Veränderungen sind die übermäßig ausgeprägte Hypertrophie der protoplasmatischen Gliazellen im motorischen Gebiet (siehe Abb. 5, 6, 7, 8, 9, 10). Abgesehen von der individuellen Disposition zur Choreainfektion, erwähnt Orzechowski, daß im Thalamus das Gewebe bei jugendlichen Individuen gliaarm sei, folglich könnten die so nahe den Capillaren direkt anliegenden Ganglienzellen, mit den Bakterientoxinen, die in der Blutbahn kreisen, in Verbindung geraten und folglich wäre die Vereinigung von Toxinen und Receptoren der Eiweißmoleküle der Ganglienzellen eine sehr intensive. Die Gliazellen und das Gliagewebe sind auch ein schützender Filter gegen Bakteriengifte. Man könnte denken, daß es in Thalamus eben wegen der speziellen, konstitutionellen schwächeren Entwicklung des Gliagewebes aber überhaupt im motorischen Gebiet zu einer bedeutenden Hypertrophie des Gliagewebes kommt, ebenso wie es in vielen Fällen der Infektion zur Hyperplasie und Hypertrophie des Drüsengewebes kommt (Status lymphaticus, hypoplasticus). Die Staphylokokken haben sowieso eine ausgesprochene große Affinität zum Zentralnervensystem, während sich beim Staphylococcus sepsis nur eine geringe gewebliche Reaktion findet. So fand ich bei mit Chorea einhergehender Sepsis eine schwere reaktive Veränderung der motorischen Bahnen, die Proliferation der protoplasmatischen Glia und darauffolgende Nekrose der Gliagewebe. Es erklärt sich aus derselben Ursache, daß Hudovernig die Choreakörperchen eben in großer Anzahl in der motorischen Bahn als Ausdruck einer schweren lokalisierten Schädigung fand. Die motorische Rinde ist die erste, beste Ansiedlungsstelle der Staphylokokken.

Wenn wir nun zwischen der Paralyse, als einer allgemeinen Infektion 
der gesamten Hirnrinde und des Markes und der Chorea infectiosa, als einer spezifischen Infèktion des Gehirns eine Parallele ziehen, so können wir feststellen, daß die Spirochäten und ihre Toxine ihre Wirkung fast in gleichem Maße auf die verschiedenen Bestandteile des Zentralnervensystems ausüben, wenn auch hier große Unterschiede in der Empfindlichkeit gewisser Nervenbahnen und Kortexpartien lange schon festgestellt sind. Schaffer schreibt in einer seiner Arbeiten über die Paralyse (K. Schaffer: Hirnpathologische Beiträge. II. Bd. 1. H. Beiträge zur Histopathologie der protoplasmatischen Neuroglia): An vielen Stellen seitens des Gliagewebes ist ein aktives Verhalten festzustellen. Die Glia spielt hier keineswegs die Rolle eines Ersatzmaterials, sondern hekundet eine selbständige, eigenartige Veränderung. Es läßt sich bei der progressiven Paralyse eine sehr starke, teils protoplasmatische, teils faserige Hypertrophie, oft mit Degeneration gepaart, feststellen und es ist sehr wichtig, daß die zonale Glia im Verlauf ihrer Änderung den normalen Bau auflöst, zu atypischen, extracorticalen Wucherungen gelangt. Aus diesem Verhalten ist es klar, daß die Glia bei der Paralyse las abgestorbene Nervenparenchym nicht allein ersetzt, sondern Wucherungen ganz individuellen (harakters vollzieht und damit beweist, daß sie nicht so sehr eine akkommodierende, passive, als vielmehr eine aktive, velbständige Rolle spielt. Bei der Paralyse erkrankt mit den Nerven und Gefäßgewebe gleichzeitig und in allen Fällen auch die Neuroglia, und indem bei der Paralyse die Nervenelemente und die Gefäße von den luetischen oder postluetischen Virus krank werden, so gibt es auch keinen (rrund, zur glejchen Yeit und in derselben Weise erkrankte (dliagewebe anders geartet affiziert aufzufassen: Das Gliagewebe ist daher von derselben Noxe erkrankt, in derselben Weise wie das Nervengewebe. Die beständige Veränderung der Neuroglia bei der Paralyse deutet schon allein auf den hervorragenden histopathologischen Wert derselben. -

Nach Sch affer soll also die Glia fast überall im Zentralnervensystem primäre Veränderungen aufweisen, die durch die luetische Infektion verursacht seien. Bei der choreatischen, Staphylokokkeninfektion ist die Gewebsreaktion der Glia eine nicht so sehr ausgebreitete, sondern sie beschränkt sich auf die bewegenden Bahnen, und auch bei der Staphylokokkeninfektion ist die Veränderung der Neuroglia eine primäre, da eine aktive Proliferation des protoplasmatischen Gliagewebes in allen unseren vier Fällen nachweisbar war, dies beweisen ja die äußerst kurzen Verlaufszeiten der vier tödlichen Choreafälle. Der Hypertrophie der Neuroglia folgt noch schneller als bei der Paralyse die schwere Glianekrose und die Verfaserung der protoplasmatischen Gliazellenfortsätze (siehe Abb. 12, 13).

Die Hypertrophie und die Verfaserung der protoplasmatischen Glia- 
fortsätze zu riesig langen Weig er tfasern ist in dem Marklager am ausgesprochensten zu sehen. Bei der Chorea wie bei der Paralyse ist eine gewisse konstitutionelle Eigenart des Zentralnervensystems ein Grund mehr zur Erkrankung. Bei der Chorea infectiosa scheint die angeborene Kleinheit und Steilheit der Bindearme und des Thalamus sowie der Brücke eine Ursache der Entstehung der Chorea infectiosa zu sein. Meine diesbezüglichen Untersuchungen, die ich anderswo mitteilen werde, scheinen das zu bestätigen. Die choreatischen Veränderungen sind streng an die bewegenden Bahnen lokalisiert, und sind das Ergebnis der Reaktion des Gliagewebes auf die rheumatischen Toxine. Die Affinität der motorischen Bahn speziell deren Nervenelemente und des sie umgebenden Neurogliafilters ist eine immens große, derzufolge die Infektion zur primären Hypertrophie des protoplasmatischen Neuroglias der zur Chorea prädisponierten Individuen führt, auf der bald eine schwere Gliadegeneration folgt.

Meinem hochgeehrten Chef, Herrn Hofrat Prof. Dr. E. E. Moravosik, sei für seine oft genossenen Unterweisungen während meiner Arbeit und des regen Interesses für dieselbe an dieser Stelle wärmstens g'edankt.

\section{Literaturverzeichnis.}

Abra ms, Brit. ned. journ. 1900. - Alzhei mer, Vortrag aus der Wanderversammlung Südwestd. Neurologen 1911. - A p pert, Le journ. med. 1898. Babes - Marinesco, Zit. bei Poynton-Holmes. - Blaschko, Virchows Archiv 83. - Broadbent, The Brit. med. journ. 1869. - Ca mis a, Zentralbl. f. Bakt. 57. 1910. Orig. -- Ces ari De mel, Acad. della science di Torino 1898. Clarke, A., Brit. med. Journ. 1876. - Colli ns, Brit. med. journ. 1913. Cramer Többen, Monatsschr. f. Psych. u. Neurol. 18. 1906. - Dana, Brain 1. 1890. - Dick - Roths tei n, Neurol. Centralbl. 1915. - Dicki ns on, Lancet 2. 1875. - Donath, Zeitschr. f. d. ges. Neur. u. Psych. 4. 1911. - Elischer, Virchows Archiv 63, 61. - Forss ner, Jahrb. f. Kinderheilk. 21. 1910. - Fraenckel, E., Virchows Archiv 194. 1908. - Fröhlich, Jahrb. f. Kinderheilk. 54. 1900. - Guiderotti - Guisetti, Zit. bei Hudovernig. - H. Handford, The Brain 1889. - Heubner, Internat. Beitr. \%. inn. Med. 1. 1902. - Hudovernig: Arch. f. Psych. 1903. - Jakovenka, Neurol. Centralbl. 1889. Köster, Münch. med. Wochenschr. 1902. - Lé pi ne, Giraud Rebattu. Rev. de med. 30. 1910. - Lered de, Rev. des mal. de l'enfance 1891. - Maragliano, Zentralbl. f. inn. Med. 1899. - Me yer, Zeitschr. f. klin. Med. 46. 1902. - Heinrich Meyer, Jahrb. f. Kinderheilk. 40. 1895. - Meinert, Allg. Wiener med. Ztg. 1868. - Mircolli: Berl. klin. Wochenschr. 1900, Nr. 19. - B. Na unyn, Mitt. a. d. med. Klin. zu Königsberg 1888. -- Nauwer k, Zieglers Beitr. z. allg. Path. u. pathol. Anat. 1. 1886. - Meisser, Handb. f. pathog. Mikroorg. 4. Ne urath, Ergebn. d. inn. Med. 9. - Ogle, Brit. for. med. chir. Red. 1868. -Okada, Mitt. a. d. med. Fakult. zu Tokio 6. 1903. - Oppenheim-Hoppo, Arch. f. Psych. 25. - Orzechows ki, Arbeiten a. d. Neurol. Inst. d. Wienes Univ. 16. 1907. - Oseki, Zieglers Beiträge \%. all. Path. u. pathol. Anat. 52. 1912. - Pianes e, Ref. Neurol. Centralbl, 1893. - L. Po poff, Virchows Archiv 63. 
352 J.Schuster: Beitrag zur Histopathologie u. Bakteriologie der Chorea infektiosa.

- Powell, Brit. med, journ. 1889. - Poynton-Holmes, Lancet 2. 1906. - Poynton-Paine, Lancet 2. 1900. - Poynton-Paine, Lancet 2. 1905. - Preobrajensky, Ref. Neurol. Centralbl. 1902. - Reichard, Dtsch. Arch. f. klin. Med. Y2. 1902. -- Reinhold, Dtsch. Zeitsehr. f. Nervenheilk. 13. 1898. - Richards, Journ. of the Amer. med. assoc. 62. 1914. - Rindfleisch, Dtsch. Zeitschr. f. Nervenheilk. 23. 1903. - R unge, Arch. f. Psych. 46. 1910. Sachs, The med. Rec. 73. 1908. - Sand, Journ. de neurol. 1912. - Savada, Virchows Archiv 166. 1901. - Schwarz, Pester med.-chir. Presse 1887. Thoms on, Brit. med. Journ. 1899. - Tomasi Crudeli, Riv. sperim. di Freniatria 34. 1908. - Triboulet, Rev. des malad. de l'enfance 1. 1891. - Wadsack, Beitr. z. klin. Med. 1904. - E. Walker, Brit. med. journ. 1907. - Weill Gallavardin, Rev. mens. des malad. de l'enfance 19. 1901. - Weleminsky, Prager med. Wochenschr. 16. 1891. - Wendenburg, Monatsschr. f. Psych. u. Neur. 28. 1910. - Werner, Münch. med. Wochenschr. 1899. - WestphalWassermann.Walkoff, Berl. klin. Wochenschr. 29. 1899. - Wichmann, Zit. von Wollenberg. - Wollenberg, Arch. f. Psych. 23. - Wollenberg, Nothnagels spez. Path. u. Ther. 12. - Ziemssen, Handb. d. spez. Path. 12. 